\title{
Gobernanza universitaria en tiempos de crisis sociosanitaria: experiencias de directivos chilenos
}

\author{
ALEX VÉLIZ BURGOS* \\ ANITA DÖRNER PARIS** \\ ALEXIS SOTO SALCEDO*** \\ ALVARADO TOLEDO****
}

Artículo de investigación sobre los retos que deben enfrentar los equipos directivos en tiempos de crisis sociosanitaria.

Recibido: 27 de abril de 2020 - Evaluado: 23 de julio de 2021 - Aceptado: 15 de septiembre de 2020

Citar como: Véliz, A., Dörner, A., Soto, A. y Alvarado, P. (2021). Gobernanza universitaria en tiempos de crisis sociosanitaria: experiencias de directivos chilenos. Hallazgos, 18(35), 31-53. Dor: https://doi. org/10.15332/2422409X.5454

* Doctor en Psicología. Académico e investigador del Departamento de Ciencias Sociales, Universidad de Los Lagos, Chile.

Correo electrónico: alex.veliz@ulagos.cl

ORCID: https://orcid.org/0000-0003-1371-9041

** Magíster en Educación Universitaria. Académica del Departamento de Salud, Universidad de Los Lagos, Chile.

Correo electrónico: anitapatricia.dörner@ulagos.cl ORCID: https://orcid.org/0000-0002-5089-8168

*** Magíster en Educación. Académico de la Escuela de Psicología Temuco, Facultad de Ciencias, Universidad Mayor, Chile.

Correo electrónico: alexis.soto@umayor.cl ORCID: https://orcid.org/0000-0002-1304-4438

**** Magíster en Ciencias de la Educación. Académica del Departamento de Humanidades y Artes, Universidad de Los Lagos, Chile.

Correo electrónico: palvarado@ulagos.cl

ORCID: https://orcid.org/0000-0002-4022-7571 


\section{Resumen}

Este artículo corresponde a un estudio cualitativo que, por medio de la teoría fundamentada, buscó conocer los desafíos que deben enfrentar los equipos directivos en el contexto de gobernanza universitaria en tiempos de crisis sociosanitaria. Para ello, se realizaron entrevistas focalizadas a 12 directivos de nivel alto que ejercen actividades en diversas universidades regionales chilenas al momento de estallar la crisis por la covid-19, y que debieron modificar el trabajo presencial administrativo y académico presencial por la modalidad teletrabajo/online. De este modo se identificaron cinco grandes categorías de análisis: preparación ante la crisis, fortalezas, dificultades, desafíos y aprendizajes derivados de la gestión en tiempos de pandemia. Por otra parte, se observan los desafíos en el área de competencias relacionales que favorecen el liderazgo y la gestión.

Palabras clave: COVID-19; Gestión universitaria; Gobernanza universitaria; Liderazgo. 


\title{
University governance in times of social and sanitary crisis: experiences of Chilean directors
}

\begin{abstract}
This article corresponds to a qualitative study that, through grounded theory, sought to understand the challenges that management teams must face in the context of university governance in times of social and sanitary crisis. To do so, focused interviews were conducted with 12 top-level managers who were working in various regional Chilean universities at the time of the crisis caused by the CoviD-19, and who had to modify their face-to-face administrative and academic work to teleworking/online form. Thus, five major categories of analysis were identified: preparation for the crisis, strengths, difficulties, challenges and lessons learned resulting from management in times of pandemic. On the other hand, the challenges in the area of relational competencies that favor leadership and management are observed.
\end{abstract}

Keywords: COVID -19; University management; University governance; Leadership.

\section{Governança universitária em tempos de crise sociossanitária: experiências de diretores chilenos}

\section{Resumo}

Este artigo corresponde a um estudo qualitativo que, por meio da teoria fundamentada, pretendeu conhecer os desafios que a equipe de direção deve enfrentar no contexto de governança universitária em tempos de crise sociossanitária. Para isso, foram realizadas entrevistas focalizadas com 12 diretores de alto escalão que exercem atividades em diversas universidades regionais chilenas que atuam durante a crise da covid-19 e que tiveram que modificar o trabalho presencial administrativo e acadêmico presencial pela modalidade de teletrabalho/on-line. Desse modo, foram identificadas cinco grandes categorias de análise: preparação ante a crise; fortalezas; dificuldades; desafios e aprendizagens derivadas da gestão em tempos de pandemia. Além disso, foram observados desafios na área de competências relacionais que favorecem a liderança e a gestão.

Palavras-chave: COVID-19; Gestão universitária; Governança universitária; Liderança. 


\section{Introducción}

Los tiempos de crisis traen periodos de acción rápida y efectiva, acompañados de momentos para reflexionar, que van apoyando procesos de ajuste para enfrentar las contingencias diarias que se presentan en las diferentes comunidades (Véliz y Dörner, 2020). Esta situación debe ser conducida en cada organización por liderazgos efectivos para hacer frente a estos procesos de desarrollo y cambio. Cada momento y cada organización requiere características especiales en las personas que ejercen el liderazgo, dentro de lo cual se destacan las habilidades directivas, un fuerte componente de habilidades sociales relacionadas con la comunicación, la cercanía, la empatía y la negociación a la hora de enfrentar conflictos (Dörner, Pereira y Arriagada, 2019).

Cuando se analizan las organizaciones de educación superior, se puede notar que estas conforman sistemas sociales complejos en los que coexisten subgrupos con características, fines y necesidades distintas, que se manifiestan por medio de diferentes objetivos: por un lado, enseñanza, expectativas de desarrollo y crecimiento desde la academia; por el otro, bienestar, estabilidad económica, sustentabilidad y proyección de la institución como objetivo común a todos los grupos (Kehm, 2012). En este contexto, quienes lideran las organizaciones deben tener presente estas dimensiones y cómo generar mayor estabilidad frente a las presiones o cambios del entorno. Tales características inciden en la gobernanza de las universidades (Schmal y Cabrales, 2018). Sin embargo, este entorno diverso es complejo dada la diversidad de las organizaciones que forman parte de lo que llamamos mundo universitario (Brunner y Pedraja-Rejas, 2017; Ganga-Contreras, Pedraja-Rejas, Quiroz Castillo y Rodríguez-Ponce, 2017).

Ahora bien, las últimas situaciones acontecidas en Chile y en el mundo han desnudado un modelo convencional de gestión que ha sido difícil de actualizar, puesto que cuando recién se iniciaba un proceso de ajuste estructural y de operación respecto a la crisis social vivida en Chile entre octubre de 2019 y marzo de 2020, surge la pandemia de la covid-19, que vuelve a instalar la incertidumbre en los procesos locales de gestión universitaria.

Este fenómeno ha despertado el interés respecto a las formas de abordaje que se están dado en las distintas instituciones para enfrentar la crisis social y la sociosanitaria, y de cómo estas formas han permitido llevar un desplazamiento más o menos fluido con sus integrantes hacia una gestión en la emergencia que permite avanzar en logros o misiones institucionales. De este modo, se han podido verificar los liderazgos que han debido ejercer los directivos de universidades para enfrentar 
la gobernanza de sus organizaciones a través del trabajo en línea, el teletrabajo y la gestión, cuando la contingencia se relaciona con la cotidianeidad de la vida de las personas y sus necesidades en tiempos de crisis sociosanitaria (Véliz y Dörner, 2020). A pesar de esto, hay características esenciales de las universidades que permanecen, en particular su papel social, su objeto de estudio: el conocimiento, la búsqueda de él, su creación, transmisión, difusión y gestión (Schmal y Cabrales, 2018).

\section{Gobernanza universitaria}

El término gobernanza aparece públicamente en los años ochenta, para señalar la necesidad de mejorar la eficacia y eficiencia de las organizaciones gubernamentales (Hollingsworth y Lindberg, 1985). Posteriormente, este concepto se extiende a diversas organizaciones que pretendan fijar marcos referenciales de comportamiento organizacional (Ganga, Abello y Quiroz, 2014).

Para Lopera (2004), la gestión universitaria constituye un conjunto de estrategias que utilizan directivos y sus cuerpos colegiados con el objetivo de garantizar la sostenibilidad institucional de las universidades. De esta manera, cada universidad adopta una identidad propia en sus formas de hacer frente a la vinculación con el entorno y sus exigencias. En este sentido, la gobernanza y la cultura organizacional de cada institución están estrechamente relacionadas e inciden en la toma de decisiones más críticas de la organización (Meléndez, Solís y Gómez, 2010).

Esta capacidad de gestionar las instituciones implica fomentar en las estructuras administrativas la incorporación de nuevas formas organizativas y nuevos métodos de gestión, a fin de dar sentido de dirección a sus comunidades, y que estén en condiciones de manejar sus entornos adversos o favorables, convirtiéndolas en factores de éxito y agentes de futuro. Se busca que la gestión considere la máxima calidad de los servicios, asegurando que la acción del gobierno universitario y la gestión administrativa sea distintiva y permita un reconocimiento social por su aporte público (Meléndez, Solís y Gómez, 2010).

De esta forma, la gobernanza debe poner el acento en cómo se toman las decisiones y quiénes las toman, además de los procesos involucrados en esta gestión, considerando los efectos económicos y sociales, sean internos y externos, tras estas decisiones (Zurbriggen, 2011). Por otra parte, Alcántara y Marín (2013) agregan como factores importantes los procesos, las negociaciones y compromisos de quienes participan en las decisiones de carácter estratégico, táctico y operativo. 
Por lo expuesto, la gobernanza universitaria se asocia a un proceso de cambio que incide en la cultura organizacional y que orienta el comportamiento de una universidad, en función de los cargos laborales, la distribución de los niveles de autoridad y los valores bajo los cuales será conducida la organización para el logro de sus objetivos en un contexto de creciente complejidad. De esta forma, la gobernanza comprende una red de componentes legales, sociales, económicos, históricos y culturales en los que las instituciones se desenvuelven y gestionan sus recursos para cumplir con los fines que les exigen: ofrecer servicios educativos de calidad e impacto (Ganga, Abello y Quiroz, 2014; Ganga, Quiroz y Fossatti, 2017; Meléndez, Solís y Gómez, 2010; Carnegie y Tuck, 2010).

De esta manera, según Brunner (2011, citado en Schmal y Cabrales, 2018), la gobernanza aplicada al modelo de gestión universitaria

[...] se asocia a la forma o método de gobernarlas, esto es, a la forma en que se toman las decisiones al interior de ellas. [...] la gobernanza es la manera en que las universidades están organizadas y son operadas internamente, y cómo se relacionan con entidades y actores externos con la finalidad de cumplir sus objetivos. (p. 827)

Por su parte, López et ál. (2011) incorporan como elementos importantes dentro de la gobernanza el liderazgo y todo lo derivado de este, la rendición de cuentas públicas y las formas de participación en las decisiones que cada organización define para los miembros de su comunidad.

Ganga, Abello y Quiroz (2014) consideran que la gobernanza universitaria está relacionada con dos aspectos centrales, tanto con la manera en que se organizan y estructuran las universidades, como la modalidad bajo la cual se gestionan y vinculan con el entorno. Por otra parte, Ibarra y Rondero (2001) asimilan gobernanza universitaria y gobernabilidad universitaria, señalando que esta involucra la capacidad de sumar a la conducción de la institución a los diferentes estamentos que conforman la organización, utilizando los mecanismos establecidos para legitimar la toma de decisiones.

Si se analiza la realidad universitaria chilena, es posible reconocer distintos elementos característicos que contribuyen a la complejidad del modelo de gestión universitario del país (Communes, 2016; Labraña y Rodríguez, 2017; Pey, 2016):

1. La existencia de una realidad social dinámica a la que la universidad debe adaptarse constantemente y que en gran medida es parte de un mercado de la educación. 
2. La competencia con otras instituciones educativas que poseen modelos organizativos diferentes y con marcos burocráticos disímiles.

3. Los diferentes modelos organizativos de las universidades, que generan distintas formas de contratación de académicos y, por lo tanto, de vinculación con las universidades, lo que lleva a la pérdida de influencia de estos en la toma de decisiones institucionales.

4. Masificación acelerada y heterogénea de la oferta universitaria desregulada, con un alto porcentaje de privatización que aumenta el endeudamiento de las familias; de este modo se genera el mercado de la educación superior.

5. Baja actualización de los estatutos universitarios, lo que propicia altos niveles de burocracia en la toma de decisiones y administración de recursos, y de este modo influye en su capacidad de competir en condiciones igualitarias con modelos universitarios privados.

La gobernanza universitaria, por lo tanto, es reflejo del modelo y de la cultura organizacional que cada institución adopte, mediados por la estructura legal disponible. En Chile el modelo universitario cuenta con diversas formas de administración. Las instituciones estatales, por su parte, tienen patrocinio financiero del Estado. Muchas de sus autoridades se escogen por sistema de elección popular y se rigen por estatuto administrativo, mientras que otras universidades no cuentan con apoyo estatal o se nutren escasamente de aportes legales que benefician a estudiantes. En estos casos, su administración está ligada a corporaciones o fundaciones sin fines de lucro, que a través de sus directorios nominan a autoridades principales, mientras que autoridades intermedias se rigen por el código del trabajo que establece procesos menos estables de permanencia en el tiempo (Brunner y Ganga, 2016). Estos modelos que coexisten en Chile impactan sin duda en los procesos de gobernanza, toda vez que la evaluación de la efectividad y eficacia de la gestión cotidiana se mide con parámetros y tiempos distintos, lo que lleva a variaciones sobre cómo abordar situaciones de crisis como la acontecida, tanto en el conflicto social como en la crisis sociosanitaria por la covid-19.

En el caso específico de la universidad estatal chilena, se requiere una gobernanza diferente a la existente en la actualidad, que se caracteriza por la presencia de autoridades sin un límite claro de reelección y bajo poder de los académicos en la toma de decisiones a través de cuerpos colegiados fuertes (Schmal y Cabrales, 2018). Por el contrario, se necesita un proceso más complejo en el que prime la meritocracia 
en la conformación de los equipos, que fomente el trabajo de equipos directivos con un propósito claro, que constantemente dialogue con los diferentes estamentos de la organización y que tenga como un valor relevante la mejora organizacional continua (Dörner, Pereira y Arriagada, 2019).

Si se analiza brevemente el papel de la universidad privada chilena, el contar con mecanismos de evaluación distintivos, es posible identificar procesos que pueden ser percibidos como más veloces, pero de igual forma, al intentar recoger principios consensuados vinculados a mecanismos de acreditación institucional, se torna estructuralmente más compleja y se arriesga a perder el dinamismo propio de organizaciones privadas. En este contexto, se pretende recoger experiencias relacionadas con las fortalezas percibidas, las dificultades encontradas, los aprendizajes obtenidos y los desafíos que quedan por abordar para crecer en la gobernanza de sistemas universitarios en tiempos de crisis, especialmente en tiempos de crisis sociosanitaria internacional derivadas de la covid-19, que obliga a las universidades a tomar la decisión de desarrollar sus procesos académicos a través de lo que en Chile se ha llamado teletrabajo o trabajo a través de redes sociales.

\section{Metodología}

El presente estudio se desarrolla mediante un paradigma cualitativo interpretativo. Se sigue la teoría fundamentada como modelo teórico metodológico de investigación. La teoría fundamentada es un método utilizado dentro del paradigma cualitativo en el que se busca, con base en los datos, revelar conceptualizaciones emergentes en el discurso de los actores, y sistematizarlas en categorías, analizando los hallazgos en un proceso de constante comparación y contrastación. Este método está diseñado para generar conceptos y teorías que se fundamentan en los datos. Permite usar datos provenientes de diversas fuentes de información, cuantitativas y cualitativas. Trasciende los métodos descriptivos y sus limitaciones en la interpretación y el modo de construir los datos (Glazer, 2002; De Souza Minayo, 2013; Polgar, 2014; Sandín, 2003; Strauss y Corbin, 2002). Estas cualidades resultan muy pertinentes en el campo de las subjetividades, con lo cual es posible develar la construcción de significados de los grupos estudiados (Silvia y Fraga, 2012; Muntaner y Gómez, 2012).

Para la recolección de información se realizaron entrevistas a través de redes sociales a doce directivos de nivel medio y superior de diferentes universidades regionales, con una matrícula promedio de 10000 estudiantes y 500 académicos (siete 
hombres y cinco mujeres, con media de edad de 43,4 años), que tenían a su cargo funcionarios académicos y administrativos. Con ellos se desarrolló un análisis del proceso de implementación de la modalidad de trabajo en línea, las fortalezas del proceso, los desafíos, las dificultades enfrentadas y los aprendizajes obtenidos. Se examinó el proceso que les ha tocado liderar en sus diferentes unidades académicas durante el primer mes de inicio de su gestión, desde el primer día de suspensión de actividades presenciales en sus casas de estudios y el desarrollo de actividades de teletrabajo con sus colaboradores. Las entrevistas fueron realizadas en marzo y en la primera quincena de abril de 2020, a través de la plataforma Meet. Tuvieron una duración promedio de 40 minutos.

Se realizó un análisis de contenido de las respuestas de los directivos, de acuerdo con lo planteado por la teoría fundamentada. Se consideró la participación voluntaria y se establecieron los resguardos éticos para asegurar la confidencialidad de la información personal de los respondientes.

\section{Resultados}

En función de las respuestas dadas por los entrevistados, se pueden identificar cinco grandes categorías: 1) preparación del proceso, 2) fortalezas del proceso, 3) dificultades del proceso, 4) desafíos y 5) aprendizajes.

\section{Preparación del proceso}

Se desprende de las respuestas de los directivos la existencia de un primer momento que se puede calificar de preparación del proceso de trabajo virtual. En este proceso se desprende lo que significó gestionar la preparación de la suspensión de actividades laborales presenciales y el cambio por actividades a distancia para mantener las actividades académicas de las unidades de la universidad.

Al no ser un proceso planificado, la preparación del teletrabajo fue abrupta, puesto que el avance de la pandemia llevó a pasar de días en que se apostaba por un proceso de contención focalizado en la zona metropolitana chilena a una estrategia nacional de confinamiento. De esta manera, la preparación se dio dependiendo de las características particulares de cada líder con su equipo de trabajo en algunos casos (ET1).

Se planificó luego de estar en casa confinado (ET2). 
Las nubes y sistemas de almacenamiento remoto facilitaron el acceso a información importante (ET3).

En algunos casos se requirió visitas por turnos para cargar información en ordenadores personales y trasladar material a domicilio (ET4).

En algunos casos menores se dispuso de equipamiento y conexión específica para funcionarios claves en la comunicación de las unidades (ET5).

En nuestro caso se hizo una distinción entre labores académicas, labores administrativas y labores de soporte, generando turnos de trabajo según perfil y competencia de cada funcionario. En todos los casos la preparación y planificación han debido ajustarse, puesto que al desconocer los tiempos de cuarentena se han ido presentando requerimientos no previstos, lo que ha llevado a generar soluciones creativas que respondan a dichas necesidades emergentes (ET8).

Se organizan en un tiempo breve una serie de capacitaciones para utilizar la nueva plataforma virtual a cargo de académicos con experticia en educación virtual (ET9).

En otra área se identifican unidades que cobran relevancia en la preparación de las organizaciones para el trabajo en línea.

En el caso de nuestra universidad, cobran protagonismo la Dirección de Informática; Dirección de Docencia; Dirección de Acceso, Equidad y Permanencia (se ocupa de la logística y conectividad de los estudiantes) y desarrollo organizacional (se ocupa de ordenar las capacitaciones de los profesores) (ET4).

La dirección de docencia debe ser muy precisa al alimentar la nueva plataforma, colocando énfasis en los tres procesos claves de cada inicio de semestre: 1) carga académica, 2) proceso de matrícula e inscripción de asignaturas y 3) preparación de la enseñanza. Para este último punto, y dada la contingencia, se creó una mesa pedagógica y se solicita a las jefaturas de carrera nombrar un usuario líder que pueda hacer de mediador para canalizar los problemas de conectividad y capacitación de los profesores (ET4).

Cobran relevancia áreas que históricamente se consideran auxiliares o de apoyo como informática, asuntos estudiantiles, comunicaciones... (ET5). 
Se hizo necesario el despliegue de un equipo humano, quienes de manera telefónica se contactan con los estudiantes que han manifestado problemas de conectividad, cuyas nominas son remitidas por sus jefaturas de carreras para atender sus necesidades y en algunos casos se ha llegado hasta sus domicilios para entregar un chip o bam (ET7).

Como se observa en las respuestas de los directivos, la preparación del proceso de pasar de un modelo de trabajo presencial o tradicional a uno preferentemente en línea tuvo reacciones y acciones heterogéneas según cada organización, incluso dentro de cada departamento en cada organización. En algunos casos se planificó considerando los estamentos de cada organización. En otras organizaciones se optó por criterios de tipo económico para organizar los trabajos. En algunas, primó el criterio pedagógico. No se observan políticas y planes comunes entre directivos que indiquen coordinación entre las organizaciones para enfrentar situaciones que vayan más allá de una resolución institucional.

\section{Fortalezas del proceso}

Cuando se consulta a los directivos respecto a las fortalezas que han logrado visualizar después de un mes de trabajo mediante la modalidad a distancia, ellos señalan que han podido identificar lo siguiente:

- Reconocimiento de las posibilidades que brinda la tecnología: "Uso de tecnologías de información y comunicación” (ET1); “Oportunidad para disminuir brecha tecnológica tanto académica como estudiante" (ET2); "Posibilidad y mejorar las herramientas de educación virtual” (ET3); “Abre una línea de capacitación y formación en esta línea para los académicos” (ET6).

- Visualización de fortalezas como el reconocimiento de habilidades directivas:

"Estilos de liderazgo de cercanía y diálogo que ayudan a enfrentar la emergencia” (ET4); "Oportunidad de ejercer liderazgo distributivo por las condiciones de la pandemia” (ET9); “Mayor comprensión de procesos de autocuidado sanitario" (ET11).

- Reconocimiento de las diferencias de género como una fortaleza para enfrentar el proceso de crisis: "Diversidad de roles que cumplen habitualmente las mujeres” (ET7); “Adaptación rápida para el cambio por parte del equipo directivo, se ha demostrado la capacidad de trabajo colaborativo y una excelente disposición de 'escucha efectiva' por parte del equipo" (ET12); "La comunicación con las autoridades de manera virtual- sincrónica permite consensos y toma de decisiones efectivas" (ET5); "Importancia que cobra el rol de las mujeres del equipo directivo, 
quienes son las que dialogan y realizan mediaciones necesarias para solucionar conflictos emergentes” (ET6); “Confianza y apoyo a quienes lideran los procesos de manera centralizada y para quienes están en la operación en los campus y sedes" (ET8).

En estos relatos se observa que aparecen los recursos humanos como fortaleza del proceso. Especialmente se destaca la importancia del liderazgo organizacional para enfrentar momentos de crisis, la flexibilidad ante situaciones cambiantes, el despliegue de las habilidades socioemocionales para entablar acuerdos, fomentar el autocuidado de los equipos y su creatividad.

\section{Dificultades del proceso}

Respecto de las dificultades que han logrado visualizar durante este periodo los directivos señalan, como primera dificultad, la diversidad de las estructuras organizacionales, lo que se traduciría en estilos directivos, liderazgos y toma decisiones mayoritariamente situacionales que influyen en las acciones desarrolladas para enfrentar los primeras decisiones derivadas de la pandemia en el contexto educativo: "Estilos organizacionales matriciales que dificultan la toma rápida de decisiones” (ET1): “Excesiva burocracia institucional que dificulta la gestión” (ET4); “Resistencia al cambio tanto académica como estudiantil relacionado a la valoración de la calidad" (ET5).

Se menciona, por otra parte, la comunicación dentro de la organización como un problema de importancia en la resolución de problemas: "Fallas en la comunicación que se hacen evidentes a la hora de no existir la presencialidad” (ET7); "Resolver temas de comunicación efectiva y oportunidad de la información entre las direcciones y jefaturas entre campus y sedes" (ET9).

Los comportamientos sexistas son mencionados como una dificultad en la gestión de personas: "Machismos y micromachismos que afectan la relación entre directivos” (ET10); “Se valoran más las opiniones de los directivos masculinos porque se los considera con mayor experiencia para tomar decisiones” (E12).

Hay alusión a las características de los equipos de trabajo, la motivación y su capacidad de adaptación a los contextos nuevos: "Los equipos que dificultan la adecuada distribución de tareas por actitudes complacientes o inflexibles" (ET1); "Problemas de conexión y equipamiento de líderes y sus equipos, lo que complejiza una relación virtual” (ET5).

Por otra parte, se muestran actividades de gestión organizacional ante tiempos de crisis, lo que se observa en los siguientes testimonios: “Cambios permanentes 
del contexto de emergencia, lo que impide tomar decisiones a mediano plazo" (ET1); "Inexperiencia en teletrabajo, lo que impacta en una adecuada convivencia de la vida personal, familiar y laboral” (ET9).

Respecto a los procesos laborales y familiares derivados de procesos de adaptación al nuevo contexto laboral, sobresale lo siguiente: "Adaptación al teletrabajo y el impacto con el quehacer en el hogar, la doble presencia se intensifica y el estrés que ocasiona para tener un buen desempeño y además organizar las diferentes tareas en casa, educación de los hijos, cuidado de las personas adultas mayores, etc.” (ET3).

Una de las dificultades que expresan los entrevistados tiene relación con actitudes y conductas que revelan una cultura machista en la gestión universitaria:

Los directivos varones tienden a desconocer o minimizar el rol de las directivos mujeres... en donde se ejemplifica los micromachismos propios de esa área del conocimiento, que en este contexto se hace más evidente (ET3).

Escaza delegación de responsabilidades a las mujeres del equipo directivo en la "labores de negociación” con estudiantes; siempre se envían a los directores centrales varones que poseen mayor experiencia en la resolución de conflictos... Se utiliza la frase... "para que nos ayuden o protejan" (ET6).

Algunas jefaturas... especialmente las lideradas por varones, ignoran instrucciones emanadas por la dirección académica, para lo cual se debe acudir a instancias superiores... para que atiendan a los requerimientos que realiza (ET9).

En estas circunstancias, se apela al rol ejecutivo versus lo emocional que tienen los directivos hombres (ET11).

Se identifican problemas como las resistencias de los diferentes integrantes de la comunidad universitaria respecto a la implementación de la formación en línea por un periodo de tiempo acotado:

Algunos grupos de académicos que no están familiarizados con el uso de las tecnologías de información, quienes requieren mayor acompañamiento para las plataformas (ET1).

Estudiantes trabajadores y padres, que no manejan plataformas y han pedido inducción (ET4). 
Tantos estudiantes como académicas que son madres o que tienen familiares a cargo han manifestado que les cuesta compatibilizar el cuidado de los hijos o familiares, el estudio o trabajo y las labores del hogar (ET6).

Grupo de estudiantes que desean realizar paro on line, principalmente porque exigen rebajas de aranceles por considerar que las clases virtuales no tendrán la misma calidad que las presenciales. Además quieren contar de manera casi inmediata con equipos que les permitan conectividad, lo cual les genera mucha ansiedad, así como también que algunas carreras jamás han utilizado las plataformas de la universidad ni los correos institucionales que hoy son exigidos para el ingreso a las clases virtuales (ET8).

Problemas en la interpretación de las comunicaciones escritas y orales (ET9).

Cuando se abordan las dificultades del proceso de cambio de trabajo presencial a uno desde casa, los participantes del estudio identifican que no existió un proceso planificado, sino más bien una serie de acciones que en su mayoría fueron reactivas, en las que el manejo de la incertidumbre es un factor importante.

\section{Aprendizajes logrados}

Cuando se consulta a los directivos respecto a los aprendizajes más relevantes logrados en este primer periodo, se mencionan como importantes el reconocimiento de la interdependencia de las diferentes unidades de la organización para un adecuado funcionamiento organizacional: "La necesidad de dependencia de otras unidades para obtener resultados laborales" (ET1); "La importancia de avanzar de forma equivalente en capacitaciones y formación del equipo para enfrentar adecuadamente los conflictos” (ET3).

Se identifica la importancia de preocuparse por el bienestar de cada integrante de la organización: "La necesaria flexibilización curricular a la hora de abordar las resistencias que se presentan en miembros del equipo a la hora de transitar hacia el teletrabajo" (ET4); "El respeto a los tiempos y características de cada trabajador como parte de una familia” (ET12); "La importancia de la salud mental” (ET6); "Los vínculos que existen entre el desarrollo y estabilidad económica y el desarrollo productivo, que se hace evidente en periodos de crisis" (ET4).

Además ocurren procesos de aprendizaje institucional que son relevantes; "La priorización de objetivos estratégicos” (ET2). 
Los entrevistados también hacen referencia a los aprendizajes de las institucionales en las que laboran: "Las normas y reglamentos se pueden modificar y no son estancos... Deben adaptarse a las circunstancias" (ET2); "Se requiere programación con objetivos, metas y fechas claras para bajar los niveles de incertidumbre que se viven en el día a día” (ET4); “Gestionar reuniones más breves, pero con definición de tareas, roles, y supervisión de lo acordado” (ET7); “Dar retroalimentación positiva de manera constante a los equipos de trabajo; entender que cada uno está dando lo mejor de sí para sobrellevar esta nueva modalidad de trabajo" (ET11); "Para la comunicación el poder de síntesis es muy importante en estos tiempos de tanta información. Se requiere utilizar lenguaje adecuado y preciso" (ET12); "La flexibilidad de pensamiento y fluidez de ideas para generar las trasformaciones necesarias tanto en la gestión académica como en la preparación de la enseñanza” (ET9); “Aprender a ser más humano... Preguntar cómo te sientes, cómo está tu familia, es importante el aquí y ahora, valorar el presente" (ET12).

Se pueden reconocer varias dimensiones de aprendizajes. El más importante para los directivos es la comprensión de la importancia de las relaciones interpersonales y el despliegue de sus habilidades socioemocionales en la gestión organizacional.

\section{Desafíos}

Respecto a los desafíos que han debido enfrentar en el inicio del proceso de gestión universitaria posteriores a la decisión de cambio de modalidad de trabajo universitario, producto de la crisis sociosanitaria, los entrevistados señalan que los más importantes tienen relación con el aprovechamiento de los medios tecnológicos: "Capacitar en usos de herramientas metodológicas para asegurar el logro de aprendizajes académicos" (ET4); "Como aprovechamos esta oportunidad para fortalecer competencias tecnológicas y de docencia on line” (ET6); "Educar a los estudiantes y profesores en las ventajas y fortalezas de la educación virtual” (ET9).

Se destaca la necesidad de desarrollar estrategias de mejoramiento en la gestión comunicacional: "Importantes desafíos para la educación superior en términos de la búsqueda de estrategias de comunicación efectiva con toda la comunidad universitaria" (E2); "Construir comunidades de aprendizaje multidiversas que puedan ser usadas de forma permanente y no generen resistencias” (ET12).

En cuanto a la gestión de recursos humanos, se plantean desafíos tendientes a potenciar a las personas que forman parte de la organización: 
Generar programas permanentes de bienestar emocional y de salud de los trabajadores (ET1).

Estrategias que permitan no despersonalizar el trabajo en equipo y la relación de personalidad o de apego con las personas en este contexto del teletrabajo (ET3).

Cómo generar estrategias de salud mental de manera sistemática en estos tiempos de crisis (ET6).

El uso de tecnologías permite mayor interacción entre pares y estudiantes y paradójicamente mayor necesidad de comunicación sincrónica (ET7).

Establecer sobre todo las confianzas necesarias con los estudiantes de primer año, en atención al perfil de los estudiantes de la Universidad, para que nos abran sus micrófonos y sus cámaras y dejen el chat, ya que hasta este último ha sido su medio principal de comunicación (ET9).

Renovar las clases, sacar mayor provecho a las tecnologías y ajustar los tiempos de trabajo sincrónico y asincrónico (ET10).

Trabajo mayormente coordinado entre profesores de las carreras para generar sinergias en los productos de evaluación; se podría dar más énfasis a las metodologías de proyecto que requieren un trabajo colaborativo de varias asignaturas, lo que puede favorecer el aprendizaje integral de los estudiantes y superar los estancos o casillas de cada asignatura (ET11).

Las formas de evaluación también deberían ser modificadas considerando lo dicho en el punto anterior (ET5).

Cautivar a los estudiantes que no confían en esta modalidad, sobre todo en carreras que tienen clases mayoritariamente prácticas en sus planes formativos (ET12).

Mejorar los mensajes que se quieren trasmitir de manera clara y precisa, utilizando elementos icónicos que ayuden tanto en el lenguaje oral y escrito (ET2).

Replantearse lo que significa una estructura matricial, ya que si bien la Universidad declara una organización en esa línea, hoy se requiere urgentemente establecer claramente la dinámica, los flujos de procesos de manera que todos y todas entendamos nuestras funciones. Esto debido a que algunas jefaturas y direcciones, al encontrar problemas los traspasan y no se hacen cargo de sus propios procesos (ET5). 
El gran desafío en estos tiempos de cuarentena y el trabajo online es mantener la comunicación sistemática, pero sin saturar con tareas e información. Muy importante escuchar las necesidades de todos los estamentos de la comunidad académica y dar instrucciones claras, sencillas y estrategias que tengan foco en la salud mental y en la afectividad de las personas (ET12).

En función de las respuestas se observa que el gran desafío para las instituciones de educación superior chilena lo constituye el paso de un modelo educativo tradicional, que se fundamenta en una gestión académica y en el ejercicio docente centrado en la formación presencial, a uno mixto, que involucra componentes presenciales y una educación que emplee los recursos tecnológicos para permitir que el modelo educativo funcione en un entorno virtual, a distancia, modelo que ya es usado por muchas universidades a escala mundial. Para ello es esencial un recurso humano capacitado y motivado, con liderazgos que se adaptan a los contextos sociohistóricos.

\section{Conclusiones}

La situación de emergencia trae por sí sola una situación de incertidumbre que obliga a replantear metas y objetivos institucionales. Requiere en sí misma, por el papel que les compete a las entidades de educación superior, una estrecha relación con la comunidad, pues esta espera que a través de la Universidad se den espacios de contención, propuestas de mejora y estrategias para abordar la conflictividad social que desata esta crisis. La complejidad que enmarca al conflicto y sus tipologías sugiere que el ser humano replantee su posición frente a este y no estigmatice este concepto como algo nocivo por excelencia, sino que, por el contrario, visualice su trasfondo y con ello las posibilidades que ofrece para el desarrollo social (David, 2019). En este contexto se avanza hacia un liderazgo que se desplaza desde adentro hacia afuera, y en el que las decisiones del gobierno universitario no solo impactan a la comunidad interna, sino también a los territorios y lugares donde se insertan. De este modo se complejiza el efecto que cada una de las acciones y decisiones traen consigo, lo que aumenta, así, la necesidad de liderazgos democráticos que fomenten la toma de decisiones proactivas (Virgili, Ganga y Figueroa, 2015).

Por esta razón, y en virtud a que la gobernanza universitaria es un constructo multidimensional y sistémico, se plantea el desafío de contar con equipos directivos 
que tengan competencias para coordinar y motivar el trabajo con otros. La selección de los equipos directivos de las universidades debe estar vinculada a un modelo riguroso de selección, a través de procesos de reclutamientos internos o externos, y debe contar con planes de apoyo o acompañamiento para el desarrollo de estos equipos directivos. Tales directivos deben adquirir competencias que los transformen en líderes, capaces de conducir la organización en tiempos de crisis. Entre las características que deben desarrollar están las habilidades sociales, la capacidad de trabajo en equipo y la inteligencia emocional (Dörner, Pereira y Arriagada, 2019). Con ello se justificaría plenamente el vínculo que debe existir con el liderazgo y sus correspondientes características, implicancias y dimensiones.

En complemento con lo anterior, los directivos de la alta gerencia universitaria deben tener conciencia de que además de las características de liderazgo, deben asumir una serie de tareas - tanto dentro como fuera de la institución- asociadas a la planificación, comunicación, motivación, concertación política y legitimación (Ganga-Contreras, Suárez-Amaya y Alarcón, 2018). Además, es necesario estimular las competencias emocionales de los directivos para favorecer las relaciones interpersonales y contribuir al desarrollo de las organizaciones (Francisco, Véliz y Carreón, 2018). Por otra parte, es importante que los directivos comprendan el papel de las universidades y su contribución al desarrollo del bienestar de la comunidad en la que están insertos (Véliz, Soto y Dörner, 2017).

Así mismo, como lo señalan Brunner y Ganga (2016), existe la necesidad de establecer un modelo regulatorio único para la universidades chilenas, puesto que conviven marcos legales y de gestión para universidades estatales, privadas tradicionales y privadas, de forma que un modelo de gestión de la educación superior facilite las coordinaciones y acciones para cada organización que enfrenta la problemática en un contexto normal, pero sobre todo en un contexto que requiere acciones extraordinarias en momentos de crisis.

Considerando lo anterior, se deben establecer requisitos basales mínimos para cumplir en todas las universidades, entre ellos un adecuado ambiente o ambiente saludable. Es deseable para las organizaciones contar con ambientes favorables para estudiar y trabajar, ambientes positivos no solo incentivan el aprendizaje, sino que construyen relaciones fructíferas que reconocen el trabajo individual y permiten el crecimiento personal y profesional de cada uno (Soto, Dörner, García y Hernández, 2018). No obstante, es necesario el abordaje multidisciplinar del tema para encontrar el bienestar de las organizaciones y de las personas en todas sus dimensiones (Sánchez-Vázquez y Sánchez-Ordóñez, 2019; Sánchez, 2018). 
La felicidad laboraly, por ende, los ambientes positivos que favorecen la salud de una organización serían un factor clave para acelerar procesos creativos e innovadores desarrollados por las personas dentro de las universidades. En este contexto, los liderazgos transformacionales son imprescindibles para fomentar la participación de la comunidad universitaria en pos de un mejor desarrollo organizacional (Ravina, Villena y Gutiérrez, 2017; Harter et ál., 2010). Por último, y no menos importante, se requieren políticas eficientes de igualdad de género que promuevan liderazgos que consideren la posibilidad de que directivos y directivas de organizaciones puedan desarrollar sus propuestas de trabajo en un entorno que evite los estereotipos de género y que, por otra parte, promueva el desarrollo y despliegue de las habilidades directivas de hombres y mujeres en un marco de respeto por las capacidades de cada liderazgo.

En este mismo sentido, la adaptación, el cambio y la permanencia de los equipos humanos son esenciales para prestar atención a aquello que nos humaniza. Cuando se reflexiona en torno a cuál debe ser la función principal de los directivos, además de la gestión, es posible encontrarse con que una de sus funciones principales es ejercer el liderazgo. Este liderazgo, entre otras acciones, debe promover y dar espacio a la comunidad educativa para que reflexione de manera profunda sobre dónde está, cómo ha llegado allí y hacia dónde quiere ir en el futuro, con lo que se fortalece así la función de la gestión directiva (Francisco y Véliz, 2020).

\title{
Sobre los autores
}

\begin{abstract}
Alex Véliz Burgos. Psicólogo. Doctor en Psicología por la Universidad del País Vasco. Académico del Departamento de Ciencias Sociales de la Universidad de Los Lagos (Ulagos). Jefe del Programa de Magíster en Salud Colectiva (Ulagos). Sus áreas de interés son los estudios multidisciplinares del bienestar humano y la salud colectiva. Entre sus más recientes publicaciones se encuentran los siguientes artículos: "Perfil de valores de estudiantes de carreras de salud del sur de Chile” (2017), "Un mundo, una salud: una propuesta de incorporación en la formación de pregrado en carreras de salud del sur de Chile” (2018), "Democracia, gobernanza y conductas éticas: ejes transversales en la formación” (2018), “Lo individual versus lo comunitario en tiempos de crisis sanitaria” (2020).
\end{abstract}

Anita Dörner Paris. Enfermera. Magíster en Educación Superior por la Universidad San Sebastián, Chile. Académica del Departamento de Salud Universidad de Los Lagos (Ulagos). Secretaria académica, magíster en Salud Colectiva (Ulagos). Sus áreas de interés son los estudios respecto a cuidados humanizados, la formación multidisciplinaria y su relación con la salud colectiva. Entre sus más recientes publicaciones se encuentran los siguientes artículos: "Hacia un modelo de universidad 
saludable: las posibilidades de implementación en una universidad estatal chilena" (2019), "Tipología de muertes de buzos profesionales ocurridas en Chile entre los años 2000 y 2012" (2018); y un capítulo de libro: "El cuidado humanizado, un desafío pendiente en la formación de profesionales en enfermería en el pregrado en el sur de Chile” (2018).

Alexis Soto Salcedo. Psicólogo. Postitulado en Psicología Educacional. Magíster en Educación. Académico y director de la carrera de Psicología, Facultad de Ciencias de la Universidad Mayor, sede Temuco, Chile. Sus áreas de interés son el bienestar psicológico, la calidad de vida, el ciclo vital (en especial familia, adolescencia y adultez mayor) y los desafíos de la educación superior. Algunas de sus publicaciones son: "El bienestar colectivo como tema de resocialización familiar en la sociedad del capitalismo informacional” (2018), "Bienestar psicológico y conductas éticas en salud” (2018), "Inteligencia emocional y bienestar psicológico en profesionales de enfermería del sur de Chile” (2017) y "El valor de la ética en profesionales del nuevo milenio: construyendo una sociedad abierta" (2019).

Paola Alvarado Toledo. Magíster en Ciencias de la Educación por la Universidad de Los Lagos, Chile. Licenciada y pedagoga en Danza de la Universidad de Artes y Ciencias Sociales (Arcis). Académica asociada al Departamento de Humanidades y Artes. Es integrante de la mesa de trabajo intersectorial de Educación Artística de la Unesco y el Mincap y de la Red Latinoamericana de Formadores de Profesores de Artes (Laifopa). Sus áreas de interés son los procesos creativos, la formación docente en artes y la educación artística. Entre sus más recientes publicaciones se encuentran los siguientes artículos: "Influencia del contexto socioeducativo en la estimulación de la creatividad: reflexiones en torno a la evaluación del proceso creativo en la formación de profesores de artes” (2018) y “Pensar y repensar la producción académica en el campo de las artes: una reflexión a partir de las experiencias docentes en una universidad al sur de Chile” (2017).

\section{Referencias}

Alcántara, A. y Marín. V. (2013). Gobernanza, democracia y ciudadanía: sus implicaciones con la equidad y la cohesión social en América Latina. Revista Iberoamericana de Educación Superior, 4(10), 93-112. https://doi.org/10.22201/iisue.20072872e.2013.10.119

Brunner, J. y Pedraja-Rejas, L. (2017). Los desafíos de la gobernanza de la educación superior en Iberoamérica. Ingeniare, Revista Chilena de Ingeniería, 25(1), 2-7.

Brunner, J. J. y Ganga, F. (2016). Gobernanza de la educación superior: una mirada crítica y propositiva al caso de Chile. Revista Dilemas Contemporáneos: Educación, Política y Valores, 4(1). Recuperado de https://www.dilemascontemporaneoseducacionpoliticayvalores. com/index.php/dilemas/article/view/182

Carnegie, G. D. y Tuck, J. (2010). Understanding the ABC of university governance. AJPA The Australian Journal of Public Administration, 69(4), 431-41. https://doi. org/10.1111/j.1467-8500.2010.00699.x 
Communes, C. (2016). El gobierno neoliberal de la universidad en Chile. De Prácticas y Discursos: Cuadernos de Ciencias Sociales, 6(8), 89-104.

David, J. Q. (2019). El conflicto social e historias de vida: la génesis del liderazgo y de la resiliencia comunitaria. Reflexiones y Saberes, (9), 58-73.

De Souza Minayo, M. C. (2013). La artesanía de la investigación cualitativa. Buenos Aires: Lugar Editorial.

Dörner, A., Pereira, R. y Arriagada, A. (2019). Hacia un modelo de universidad saludable: las posibilidades de implementación en una universidad estatal chilena. Comunidady Salud, 17(2), 60-64.

Francisco, F., Véliz, A. y Carreón, J. (2018). Enseñanza y sanación: una mirada desde una didáctica de raigambre curativa. Utopía y Praxis Latinoamericana, 23(83), 34-40. http://doi. org/10.5281/zenodo.1438526

Francisco-Carrera, F. J. y Véliz-Burgos, A. (2020). Educación, generosidad, ecología y silencio: hacia una didáctica de la salud y el bienestar en las aulas del siglo xxı. Revista Costarricense de Psicología, 39(1), 19-34. https://dx.doi.org/10.22544/rcps.v39i01.02

Ganga, F., Abello, J. y Quiroz, J. (2014). Gobernanza universitaria: una mirada histórica y conceptual. En F. Ganga, J. Abello y J. Quiroz, (Eds.). Gobernanza universitaria: aproximaciones históricas y empiricas (pp. 11-20). Santiago de Chile: Universidad de Los Lagos.

Ganga-Contreras, F., Pedraja-Rejas, L., Quiroz Castillo, J., y Rodríguez-Ponce, E. (2017). Isomorfismo organizacional (IO): breves aproximaciones teóricas y algunas aplicaciones a la educación superior. Espacios, 38(20), 31-43.

Ganga, F., Quiroz, J. y Fossatti, P. (2017). Análisis sincrónico de la gobernanza universitaria: una mirada teórica a los años sesenta y setenta. Educação e Pesquisa, 43(2), 553-68. https://doi.org/10.1590/s1517-9702201608135289

Ganga-Contreras, F., Suárez-Amaya, W. y Alarcón, N. (2018). Liderazgo en la gobernanza de las universidades: aprendiendo ciertas lecciones desde la etología. Revista Inclusiones, 5(esp.), 132-145.

Glaser, B. (2002). Conceptualization: on theory and theorizing using grounded theory. International Journal Qualitative Methods, 1(2): 23-38. https://doi. org/10.1177/160940690200100203

Harter, J. K., Schmidt, F. L., Asplund, J. W., Killham, E. A. y Agrawal, S. (2010). Causal impact of employee work perceptions on the bottom line of organizations. Perspectives on Psychological Science, 5(4), 378-389. https://doi.org/10.1177/1745691610374589

Hollingsworth, J. R. y Lindberg, L. N. (1985). The governance of the American economy: the role of markets, clans, hierarchies, and associative behaviour. En W. Streeck y P. C. Schmitter (Eds.). Private interest governments: beyond market and tate (pp. 221-267). Beverly Hills: Sage.

Ibarra, E. y Rondero, N. (2011). La gobernabilidad universitaria entra en escena: elementos para un debate en torno a la nueva universidad. Revista de la Educación Superior, 30(118), 79-100. 
Kehm, B. M. (2012). Gobernanza: ¿qué es? ¿Es importante? En B. M. Kehm (Ed.), La nueva gobernanza de los sistemas universitarios (pp. 19-43). Barcelona: Octoedro.

Labraña, J. y Rodríguez, J. (2017). Estado y universidad en Chile: problemas de distinción en torno a su función pública. Pensamiento, 54(1), 1-19. https://doi.org/10.7764/ PEL.54.1.2017.7

Lopera, P. C. (2004). Antinomias, dilemas y falsas premisas que condicionan la gestión universitaria. Revista Mexicana de la Investigación Educativa, 9(22), 617-635.

López, R., González, O., Mendoza, J. y Pérez, J. (2011). Las formas de elección de los rectores: otro camino para acercarse al conocimiento de las universidades públicas autónomas. Perfiles Educativos, 33(131), 8-27.

Meléndez, M. A., Solís, P. y Gómez, J. (2010). Gobernanza y gestión de la universidad pública. Revista de Ciencias Sociales, 16(2), 210-25.

Muntaner, C. y Gómez, M. B. (2003). Qualitative end cuantitative research in social epidemiology: is complementarity the only issue? Gaceta Sanitaria, 17(3), 53-78.

Pey, R. (2016). Autonomía universitaria y democracia. Revista Anales, 7(11), 63-80. https://doi. org/10.5354/0717-8883.2017.45229

Polgar, S. (2014). Introducción a la investigación en ciencias de la salud. Barcelona: Elsevier.

Ravina, R., Villena, F. y Gutiérrez, G. A. (2017). Una aproximación teórica para mejorar los resultados de innovación en las empresas desde la perspectiva del "Happiness Management”. Retos, Revista de Ciencias de la Administración y Economía, 14(7), 113-129. https:// doi.org/10.17163/ret.n14.2017.06

Sánchez,J. F. (2018). El significado vital en las organizaciones: aportaciones de la economía de la felicidad. Cauriensia, 13, 143-156. https://doi.org/10.17398/2340-4256.13.143

Sánchez-Vázquez, J. F. y Sánchez-Ordóñez, R. (2019). “Happiness Management”: revisión de literatura científica en el marco de la felicidad en el trabajo. Retos, Revista de Ciencias de la Administración y Economía, 9(18), 259-271. https://doi.org/10.17163/ret.n18.2019.05

Sandín, M. P. (2003). Investigación cualitativa en educación. España: McGraw-Hill/ Interamericana.

Schmal, R. y Cabrales, F. (2018). El desafío de la gobernanza universitaria: el caso chileno. Ensaio: Avaliação e Políticas Públicas em Educação, 26(100), 822-848. https://doi.org/10.1590/ s0104-40362018002601309

Silva, S. y Fraga, S. (2012). Qualitative research in epidemiology. En N. Lunet (Ed.), Epidemiology: current perspectives on research and practice (pp. 63-84). Londres: InTech.

Strauss, A. L. y Corbin, J. (2002). Bases de la investigación cualitativa: técnicas y procedimientos para desarrollar la teoría fundada. Medellín: Editorial Universidad de Antioquia.

Soto, A., Dörner, A., García, C. y Hernández, T. (2018). El bienestar colectivo como tema de resocialización familiar en la sociedad del capitalismo informacional. Utopía y Praxis Latinoamericana, 23(83), 51-56. 
Gobernanza universitaria en tiemposde crisis sociosanitaria: experiencias...

Véliz, A., Soto, A. y Dörner, A. (2017). Una propuesta multidisciplinaria para abordar salud colectiva y bienestar humano en comunidades vulnerables del sur de Chile. Revista Inclusiones, 4(2), 179-187.

Véliz-Burgos, A. y Dörner-Paris, A. (2020). Lo individual versus lo comunitario en tiempos de crisis sanitaria. Propósitos y Representaciones, 8(SPE1), e502. http://dx.doi.org/10.20511/ pyr2020.v8nSPE1.502

Virgili, Ganga, F. y Figueroa, K. (2015). Gobernanza universitaria o cogobierno: el caso de la Universidad de Concepción de Chile. Última Década, 23(42), 187-216. https://dx.doi. org/10.4067/S0718-22362015000100009

Zurbriggen, C. (2011). Gobernanza: una mirada desde América Latina. Perfiles Latinoamericanos, 19(38), 39-64. 